OPEN ACCESS

Edited by:

Satoshi Goto,

Tokushima University, Japan

Reviewed by:

Yaohui Tang,

Stanford University, United States

Bibo Tan,

Fourth Hospital of Hebei Medical

University, China

*Correspondence:

Nan Liu

xieheliunan1984@sina.com

tThese authors have contributed equally to this work.

Received: 19 June 2017 Accepted: 21 November 2017 Published: 13 December 2017

Citation:

Chen J, Du H, Zhang Y, Chen H, Zheng M, Lin P, Lan Q, Yuan Q, Lai Y, Pan X, Chen R and Liu N (2017) Netrin-1 Prevents Rat Primary Cortical Neurons from Apoptosis via the DCC/ERK Pathway.

Front. Cell. Neurosci. 11:387.

doi: 10.3389/fncel.2017.00387

\section{Netrin-1 Prevents Rat Primary Cortical Neurons from Apoptosis via the DCC/ERK Pathway}

\author{
Jianhao Chen ${ }^{1,2 t}$, Houwei Du ${ }^{1,2+}$, Yixian Zhang ${ }^{2,3}$, Hongbin Chen ${ }^{1,2}$, Mouwei Zheng ${ }^{1,2}$, \\ Peiqiang Lin ${ }^{1,2}$, Quan Lan ${ }^{1,2}$, Qilin Yuan 1,2, Yongxing Lai ${ }^{1,2}$, Xiaodong Pan ${ }^{1,4}$, \\ Ronghua Chen ${ }^{1,2}$ and Nan Liu 1,2,3*
}

'Department of Neurology, Fujian Medical University Union Hospital, Fuzhou, China, ${ }^{2}$ Institute of Cerebral Vascular Disease of Fujian Province, Fuzhou, China, ${ }^{3}$ Department of Rehabilitation, Fujian Medical University Union Hospital, Fuzhou, China, ${ }^{4}$ Key Laboratory of Brain Aging and Neurodegenerative Diseases, Fujian Key Laboratory of Molecular Neurology, Fujian Medical University, Fuzhou, China

In the nervous system, Netrin-1 serves as a neural guide, mediating the neuronal development. However, it remains blurred whether Netrin-1 can protect neurons from apoptosis induced by cerebral stroke. In the current study, the cultured rat primary cortical neurons were transfected with Netrin-1-encoding lentivirus before the oxygenglucose-deprivation (OGD) treatment. Cell death and apoptosis were evaluated by lactate dehydrogenase $(\mathrm{LDH})$ release and flow cytometry. We found that Netrin-1 attenuated OGD-induced cell death and neuronal apoptosis at $24 \mathrm{~h}$ after OGD treatment, and that the overexpression of Netrin-1 activated the ERK signaling pathway. These effects were partly abolished by blocking its receptor deleted in colorectal cancer (DCC) or U0126, an inhibitor of the ERK signaling pathway. Netrin-1 overexpression in neurons elevated the expression of DCC, on mRNA level and protein level. Netrin-1 also reduced DNA damage. Taken together, our findings suggest that Netrin-1 attenuates cell death and neuronal apoptosis via the DCC/ERK signaling pathway in the cultured primary cortical neurons after OGD injury, which may involve the mediation of DNA damage in the neurons.

Keywords: Netrin-1, primary cortical neurons, DNA damage, DCC, ERK, OGD

\section{INTRODUCTION}

Cerebral stroke often features the occlusion or hemorrhage of cerebral vessels and results in physical dysfunction or even death of the organisms. It is responsible for almost half of the acute neurological disorders. Cerebral ischemia induces a deprivation of oxygen, which can endanger neuronal survival or damage the neurons by triggering overlapped cell signaling pathways. Consequently, necrosis and apoptosis, which contribute to the neuronal death, will follow suit (Nakka et al., 2008). Therefore, protecting neurons from the ischemic damage remains a priority in treating cerebral stroke conditions.

In the nervous system, Netrin-1, as a guide in neuronal migration, attracts axons towards the middle of spinal cord, which is mediated by deleted in colorectal cancer (DCC; Forcet et al., 2002), and promotes neurogenesis in the striatal primordium (Hamasaki et al., 2001). It also plays a chemorepulsive role in axons of trochlear, trigeminal and facial nerves, which is mediated by Unc5-related proteins (Yung et al., 2015). Moreover, Netrin-1 plays a key role in angiogenesis after stroke. Studies document that Netrin-1 stimulates the proliferation 
and migration of human cerebral endothelial cells (HCECs) and human aortic smooth muscle cells (HASMCs; Fan et al., 2008; Lu et al., 2012) and that Netrin-1 overexpression in mouse brain promotes angiogenesis and long-term neurological recovery after transient focal ischemia (Fan et al., 2008; Lu et al., 2012). Recent research into tumors reveals that Netrin-1 is also involved in the inhibition of tumor cell apoptosis (Thiebault et al., 2003; Paradisi and Mehlen, 2010). In the research of Mazelin et al. (2004), Netrin-1 promoted colorectal tumorigenesis by inhibiting the cellular apoptosis. But its receptor, DCC can induce apoptosis conditionally, in which DCC acts as a dependent receptor to induce apoptosis. When DCC engaged with Netrin-1, the pro-apoptotic effect was abolished. Netrin-1 also acts as a survival factor in other research (Furne et al., 2008; Delloye-Bourgeois et al., 2009b; Harter et al., 2014). However, little literature is available to illuminate its anti-apoptotic activity in neurons that are subject to oxygen-glucose-deprivation (OGD) condition, which mimics the clinical cerebral ischemic situations.

In tumor research, Netrin-1 inhibits the ERK signaling pathway by combining with UNC5B in pancreatic ductal adenocarcinoma, indicating that the pathway is closely related to the mediation of Netrin-1 (An et al., 2016). In different pathologic conditions, the ERK signaling pathway is involved in apoptosis (Chen et al., 2015; Tan et al., 2016; Lewinska et al., 2017; Weng et al., 2017). In the research of Ma et al. (2010), one of Netrin-1 receptors, DCC, activates the ERK signaling pathway. We speculated that Netrin-1 overexpression could protect cortical neurons from apoptosis by activating the ERK signaling pathway via the mediation of DCC expression. As the ERK signaling pathway has also been documented to affect DNA damage (Dai et al., 2008; Gao et al., 2013; Lee et al., 2013; Tian et al., 2013), we wondered whether Netrin-1 may protect primary cortical neurons after OGD through reducing DNA damage.

In the current study, cultured rat primary cortical neurons were transfected with Netrin-1 encoding lentivirus and subsequently subjected to OGD treatment to mimic an ischemic condition. We found that Netrin-1 attenuated OGD-induced cell death and neuronal apoptosis and that the overexpression of Netrin-1 activated the ERK signaling pathway. These effects were partly abolished by blocking its receptor DCC or U0126, an inhibitor of the ERK signaling pathway. Additionally, Netrin-1 reduced DNA damage via the DCC/ERK pathway. Altogether, these findings suggest that Netrin-1 can improve the outcome of cerebral ischemia.

\section{MATERIALS AND METHODS}

\section{Animals}

Pregnant Sprague-Dawley rats were purchased from Animal Center of Fujian Medical University (Fuzhou, China). Animals were fed and housed in standard conditions. Animals were euthanized with isoflurane which contained 3\% induction, $1.5 \%$ maintenance in $30 \% \mathrm{O}_{2}$ and $70 \% \mathrm{~N}_{2} \mathrm{O}$. The protocols followed the guidelines of National Institute of Health (NIH Publications No. 80-23, revised in 1996). The experiments were approved by Institutional Animal Care and Use Committee of Fujian Medical University.
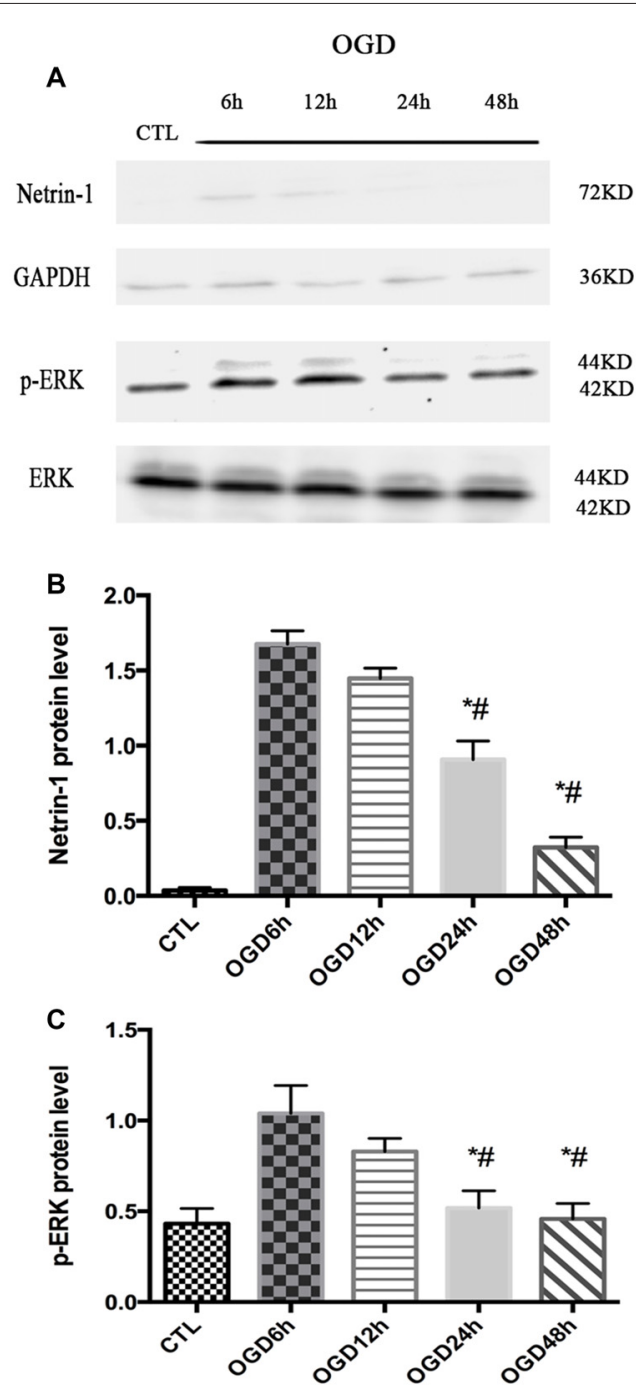

FIGURE 1 | The expression of Netrin-1 and p-ERK in oxygen-glucose-deprivation (OGD) condition across different time points. (A) The images of the result of western blot. (B) The analysis of result of western blot for Netrin-1. Data are presented as mean \pm SEM. ${ }^{*} p<0.05$, as compared with the OGD 6 h group; ${ }^{\#} p<0.05$, as compared with the OGD 12 h group; Mann-Whitney U-test; $n=4$. (C) The analysis of result of western blot for $p$-ERK. Data are presented as mean \pm SEM. ${ }^{*} p<0.05$, as compared with the OGD 6 h group; ${ }^{\#} p<0.05$, as compared with the OGD $12 \mathrm{~h}$ group; Mann-Whitney U-test; $n=4$.

\section{Primary Cortical Neuron Cultures}

Primary cortical neurons were cultured as described previously (Chen et al., 2016). Briefly, samples were prepared from embryonal brains (aged 16-18 days) of Sprague-Dawley rats. The cell suspensions were seeded on 6-well cell culture plates or coverslips $(24 \mathrm{~mm} \times 24 \mathrm{~mm})$, with poly-L-lysine coated and cultured in neurobasal medium containing $2 \%$ B27, $0.5 \mathrm{mM}$ of glutamine and $50 \mathrm{U} / \mathrm{ml}$ of penicillin/streptomycin. The harvested cells were cultured at $37^{\circ} \mathrm{C}$ with $5 \% \mathrm{CO}_{2}$. The neurobasal medium was refreshed in $8 \mathrm{~h}$ and then half of the medium was refreshed every 2 days. 
A

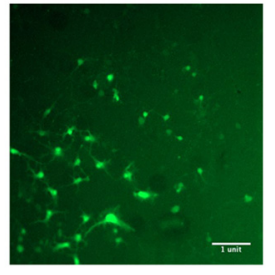

B

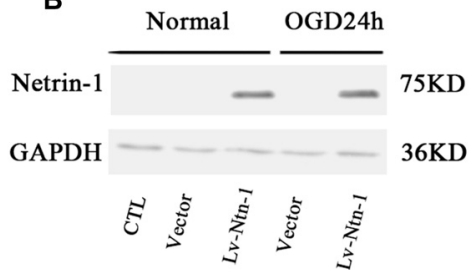

C

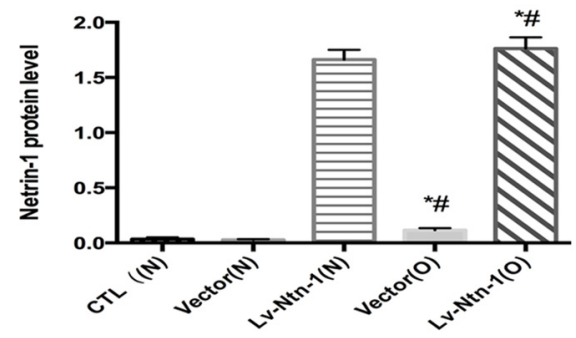

FIGURE 2 | The overexpressed Netrin-1 lentivirus transfected into primary cortical neurons. (A) The lentivirus containing gene green fluorescent protein (GFP) was transfected into primary cortical neurons. (B) The image of the result of western blot. (C) The analysis of result of western blot for Netrin-1. Data are presented as mean \pm SEM. ${ }^{*} p<0.05$, as compared with the vector (N) group; $" p<0.05$, as compared with the Vector (O) group; Mann-Whitney U-test; $n=4$.

\section{Lentivirus Transfection}

The cultured neurons were transfected with lentivirus which overexpressed Netrin-1 (the Lv-Nnt-1 group) or vector (the vector group) at an multiplicity of infection (MOI) of 1 according to the manufacturer's instruction (Genechem, Shanghai, China). Eight hours after transfection, the cultured neurons were processed for various studies. Cells transfected with lentivirus contained the gene that encodes green fluorescent protein (GFP). The transfection efficacy of lentivirus was $96 \%$.

\section{Oxygen-Glucose Deprivation (OGD)}

The OGD model was established as described previously with modifications (Chen et al., 2016). In the vector group and Lv-Nnt-1 group, 7 days after plating, the cultured primary cortical neurons were incubated with glucose-free DMEM and further incubated in an anaerobic chamber, which contained 5\% $\mathrm{CO}_{2}$ and $95 \% \mathrm{~N}_{2}$, at $37^{\circ} \mathrm{C}$ for $120 \mathrm{~min}$. Then the cultured neurons were switched back to the pre-OGD culture condition. The control group (CTL group) was not subject to OGD exposure.

\section{Drug Treatment}

For neurons that were transfected with the lentivirus that encodes Netrin-1, U0126 (CST, USA), a specific inhibitor of the Raf/MAPK/ERK signaling pathway, was added to inhibit the phosphorylation of ERK, with a final concentration of $20 \mathrm{nmol} / \mathrm{L}$ (the Lv-Nnt-1 + U0126 group). The drug was added to the medium after OGD. DCC blocking antibody $(5 \mu \mathrm{g} / \mathrm{mL}$; Santa Cruz, CA, USA) was applied to the cultured neurons of the Lv-Nnt-1 group right before and after OGD to antagonize the effect of DCC (the Lv-Nnt-1 + anti-DCC group).
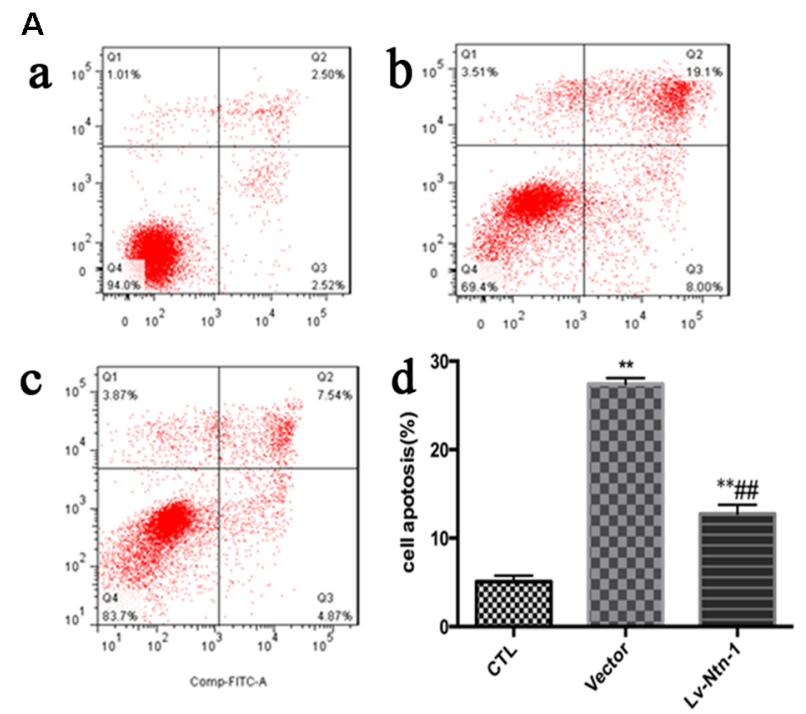

B

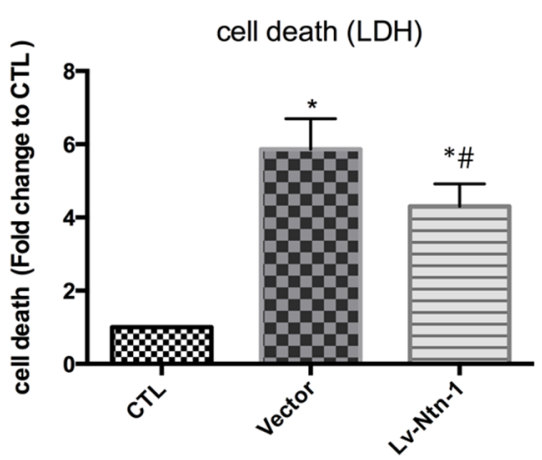

FIGURE 3 | OGD-induced cell death and apoptosis attenuated by Netrin-1 expression. (A) Twenty-four hours after OGD, cell death and apoptosis were detected by lactate dehydrogenase $(\mathrm{LDH})$ release and flow cytometry. The signals detected in Q2 and Q4 quadrants represented the apoptotic neurons. (a) Control group; (b) Vector group; (c) LV-Ntn-1 group. (d) The analysis of the result of flow cytometry. Data are presented as mean \pm SEM. ${ }^{*} p<0.05$, as compared with the control group; ${ }^{*} p<0.05$, as compared with the vector group; ${ }^{* *} p<0.01$, as compared with the control group; ${ }^{\# \#} p<0.01$, as compared with the vector group; One-way analysis of variance (ANOVA; $\left.F_{0.05(2,6)}=587.439\right) ; p<0.001 ; n=3$. (B) Cell death rates of each group, which were measured by LDH release. Data are presented as mean $\pm \mathrm{SEM}$. ${ }^{*} p<0.05$, as compared with the control group; ${ }^{\#} p<0.05$, as compared with the vector group; Mann-Whitney U-test; $n=4$.

\section{Quantitative PCR (qPCR)}

Total RNA was isolated from the cultured neurons with TRIzol according to the instructions. RNA was reversely transcripted to cDNA. The SYBR Green I qPCR kit (Takara, Japan) was used for quantitative PCR following the manufacturer's protocol. qPCR was detected in triplicate. The gene expression of each sample was normalized to the expression of GAPDH. The qPCR condition was incubated at $95^{\circ} \mathrm{C}$ for $10 \mathrm{~min}$ before 45 cycles of $95^{\circ} \mathrm{C}$ for $10 \mathrm{~s}, 63^{\circ} \mathrm{C}$ for $5 \mathrm{~s}$ and $72^{\circ} \mathrm{C}$ for $15 \mathrm{~s}$. Melting curve was analyzed at $65^{\circ} \mathrm{C}$, with the temperature increasing at a rate of $1^{\circ} \mathrm{C}$ every $10 \mathrm{~s}$ to $95^{\circ} \mathrm{C}$. The primers used for the research were as follows: Netrin-1, 5'-CCGTGGTGACCAGAGTTTGT-3' and 5'-ATCACCAGGCTGCTCTTGTC-3'; UNC5B, 5'-CGA 

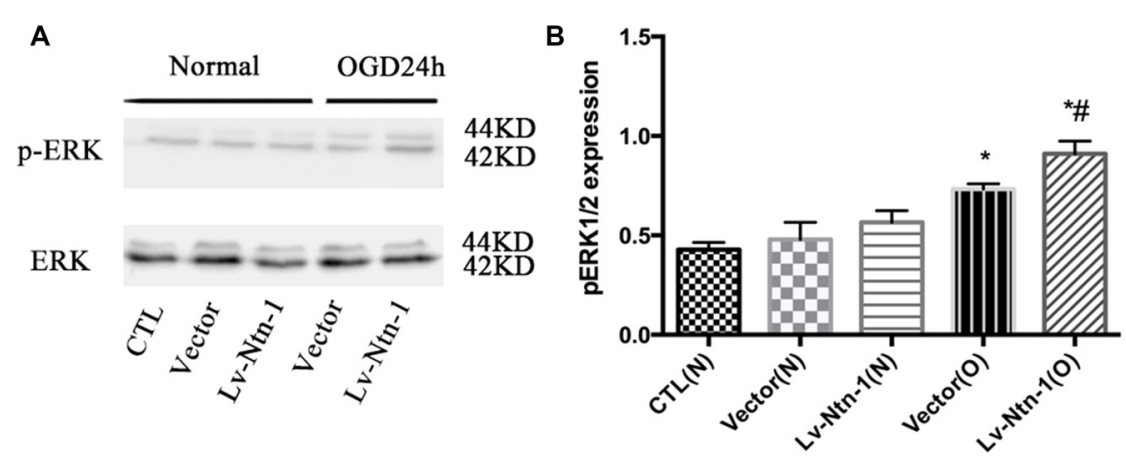

FIGURE 4 | Netrin-1 activated the ERK signaling pathway of primary cortical neurons after OGD. (A) The image of the result of western blot. (B) The analysis of result of western blot for $\mathrm{p}-\mathrm{ERK}$. Data are presented as mean $\pm \mathrm{SEM}$. ${ }^{*} p<0.05$, as compared with the vector (N) group; ${ }^{*} p<0.05$, as compared with the Lv-Ntn-1 (N) group; Mann-Whitney U-test; $n=4$.

CCCTAAAAGCCGCCCC-3' and $5^{\prime}$-GGGATCTTGTCGGCA GAGTCC- ${ }^{\prime}$; DCC, $5^{\prime}$-ACATCCGACGTTCGGCTTT- $3^{\prime}$ and 5'-TGATTTTCCCATTGGCTTCC-3'; GAPDH, 5'-CCC TTCATTGACCTCAACTA-3' and 5'-CCAAAGTTGTCA TGGATGAC- $3^{\prime}$. GAPDH was used as the reference gene and the expression of each targeted gene was analyzed by $2^{-\Delta \Delta C T}$ method. Moreover, the control group was used as a calibrator sample and was set as $1 \times$ expression of each targeted gene. Three independent experiments were performed.

\section{Western Blot}

The expressions of Netrin-1, ERK, p-ERK and DCC from the cultured neurons were analyzed by western blot after the drug treatment. Briefly, cell extracts were obtained from primary cortical neurons using RIPA lysis buffer containing $1 \mathrm{mM}$ PMSF and Protease and phosphatase inhibitor cocktail (Beyotime, China). The supernatant was centrifuged at $15,000 \times g$ at $4^{\circ} \mathrm{C}$ for $10 \mathrm{~min}$. The protein concentration was determined by BCA kit (Thermo, USA). Equal amount of total protein $(20 \mu \mathrm{g})$ from every sample was separated by $10 \%$ or $12 \%$ SDS-PAGE and subsequently transferred to PVDF membranes (Millipore, USA). The membranes were blocked with the blocking solution (Beyotime, China) for $1 \mathrm{~h}$ and then incubated with primary antibodies at $4^{\circ} \mathrm{C}$ overnight: rabbit anti-DCC antibody (1:200, Santa Cruz, CA, USA), rabbit anti-ERK antibody, rabbit anti-pERK antibody (1:400, Cell Signaling Technology, USA, respectively); Rabbit anti-Netrin-1 antibody and rabbit anti-GAPDH antibody (1:1000, Abcam, UK, respectively). After three washes with PBST, the membranes were incubated with goat anti-rabbit IgG-HRP secondary antibody (1:8000, Abcam, UK) at room temperature for $2 \mathrm{~h}$ and the signals of membranes were detected with ECL reagent kits (Beyotime, China). Band intensities were analyzed with the ImageJ software (1.46r). The relative expression levels of proteins were normalized to the appropriate internal control. Four separate experiments were conducted.

\section{Cell Viability Assessment}

Lactate dehydrogenase (LDH) leakage, as an indicator to the integrity of cell membrane, was used to assess the viability of neurons. It is based on LDH transformation of pyruvate to lactate in the presence of reduced nicotinamide adenine dinucleotide (NADH). The transformation of NADH to NAD is accompanied by a decrease in absorbance at $340 \mathrm{~nm}$ and the change in absorbance correlates with the LDH activity in the medium. LDH leakage was measured and calculated according to manufacturer's protocol of LDH assay kit (Beyotime, China). Four separate experiments were conducted.

\section{Flow Cytometry}

ANNEXIN V-APC/7-AAD Staining was employed to detect the apoptosis of neurons. Flow cytometry was performed as described previously with minor modifications (Lin et al., 2015). In brief, neurons were cultured in flasks $\left(25 \mathrm{~mm}^{2}\right)$, following the protocol described above. Twenty-four hours after OGD, AnnexinV-APC/7-AAD staining was performed in accordance with the manufacturer's instructions. The neurons were resuspended and washed three times with PBS $\left(4^{\circ} \mathrm{C}\right)$. Then the neurons were resuspended with 200 $\mu \mathrm{L}$ incubation buffer and then $5 \mu \mathrm{l}$ of Annexin- $\mathrm{V}$ labeling reagent and $10 \mu \mathrm{l}$ of 7-AAD were added into the medium. The neurons were incubated at room temperature in the dark for $15 \mathrm{~min}$. At least $1 \times 104$ cells were recorded in each sample and the result was analyzed by flow cytometry (Beckton Dickinson, USA). The experiment was repeated three times.

\section{Immunofluorescence Staining}

Twenty-four hours after OGD, imunofluorescence staining was performed to evaluate the DCC expression. Neurons were washed with PBS for three times and then fixed in $4 \%$ paraformaldehyde $(\mathrm{pH}$ 7.4) for $15 \mathrm{~min}$. Cells were incubated at $4^{\circ} \mathrm{C}$ overnight with rabbit anti-DCC antibody (1:20, Abcam, UK). After three washes with PBS, they were further incubated with corresponding secondary antibody, $\mathrm{Cy} 3$ donkey anti-rabbit 

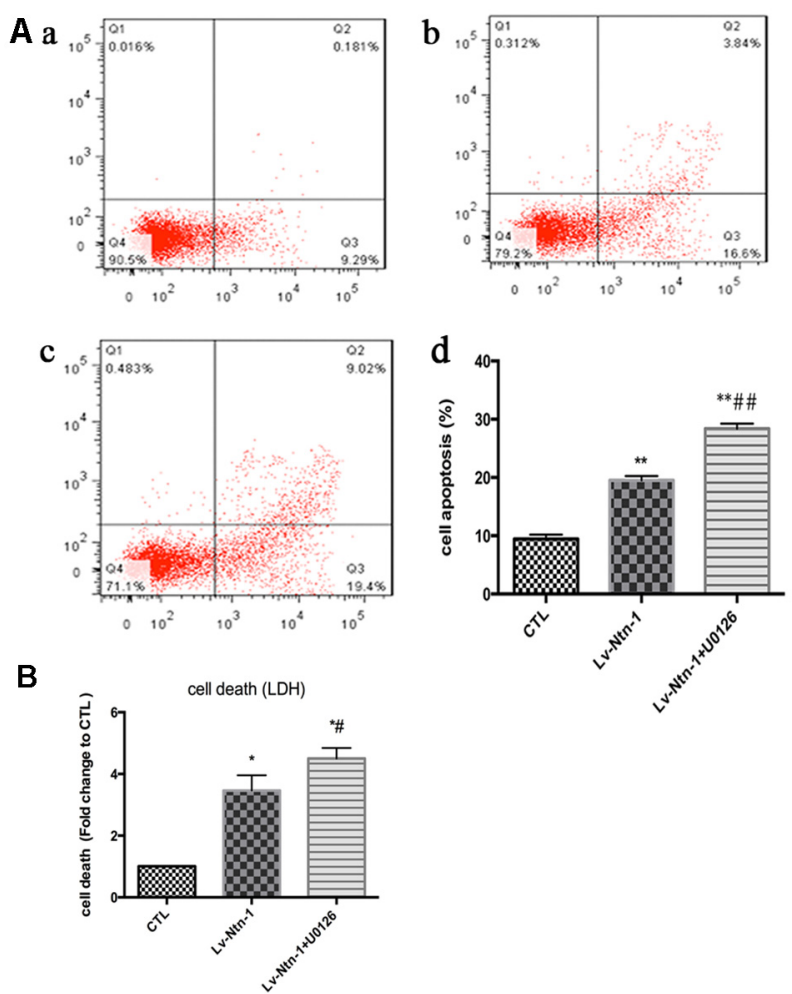

FIGURE 5 | Netrin-1-induced anti-apoptosis was abolished by the inhibitor of ERK signaling pathway, U0126. (A) The image of apoptosis detection by flow cytometry. (a) The control group; (b) The Lv-Ntn-1 group; (c) The Lv-Ntn-1 + U0126 group. (d) The analysis of the flow cytometry. Data are presented as mean \pm SEM. ${ }^{*} p<0.05$, as compared with the control group; ${ }^{*} p<0.05$, as compared with the LV-Ntn-1 group; ${ }^{* *} p<0.01$, as compared with the CTL group. $\#$ \# $p<0.01$, as compared with the Lv-Ntn-1 + U0126 group; One-way ANOVA $\left(F_{0.05(2,6)}=284.425\right) ; p<0.001 ; n=3$. (B) The analysis of cell death rates by LDH release. Data are presented as mean \pm SEM. ${ }^{*} p<0.05$, as compared with the control group; ${ }^{*} p<0.05$, as compared with the Lv-Ntn-1 group; Mann-Whitney U-test; $n=4$.

$\operatorname{IgG}(1: 400$, Jackson Immunoresearch, USA) at room temperature for $2 \mathrm{~h}$. The nuclei were stained with DAPI $(5 \mu \mathrm{g} / \mathrm{ml}$; Beyotime, USA). Glass slides were viewed under a ZEISS LSM 780 confocal microscope (Carl Zeiss, Germany), and the OD was quantified with ImageJ software as described previously. All trials were repeated three times.

\section{Comet Assay}

The comet assay was used to assess the DNA damage of neurons. After the application of coverslips, the slides were allowed to gel at $4^{\circ} \mathrm{C}$ for about $60 \mathrm{~min}$. The slides were immersed in cold lysing solution at $4^{\circ} \mathrm{C}$ for at least $1 \mathrm{~h}$, and refrigerated overnight before the alkali treatment. They underwent electrophoresis for $20 \mathrm{~min}$ at $1.6 \mathrm{~V} / \mathrm{cm}$ and $300 \mathrm{~mA}$ and then neutralization. The dried slides were subsequently stained using ethidium bromide $(20 \mathrm{lg} / \mathrm{ml})$ after appropriate fixing for $10 \mathrm{~min}$. The whole procedure was performed in dim light to minimize artifact. DNA damage was analyzed at a magnification of $200 \times$ under a fluorescence microscope (Nicon Eclips E6600, Japan). A total of 50 cells were examined per slide. The tail length and tail DNA\% were used to measure the double-strand breaks. Fifty cells were analyzed each time with the Comet Assay Software Project.

\section{Statistical Analysis}

Data were expressed as Mean \pm SEM and analyzed by SPSS 20.0 (IBM, USA). Three independent experiments were conducted for all measurements. Statistical significance among groups was determined by one way analysis of variance (ANOVA) followed by Student-Newman-Keuls multiple comparisons test when equal variances were assumed. When equal variances were not assumed, Dunnett's T3 was applied. Data are presented as mean \pm SEM. The significance of mean differences between two groups was calculated by unpaired two-tailed Student's $t$-tests. The results of western blot, cell death rate and comet assay were calculated by Mann-Whitney U-test, $P$ values less than 0.05 (two-sided) were considered as statistically significant.

\section{RESULTS}

\section{The Expression of Netrin-1 and p-ERK Was Assessed after OGD Across Different Time Points}

The expression of Netrin-1 and p-ERK was assessed after OGD across different time points ( 6 h, 12 h, $24 \mathrm{~h}$ and $48 \mathrm{~h}$ ) by western blot. The expression of Netrin-1 and p-ERK both increased at 


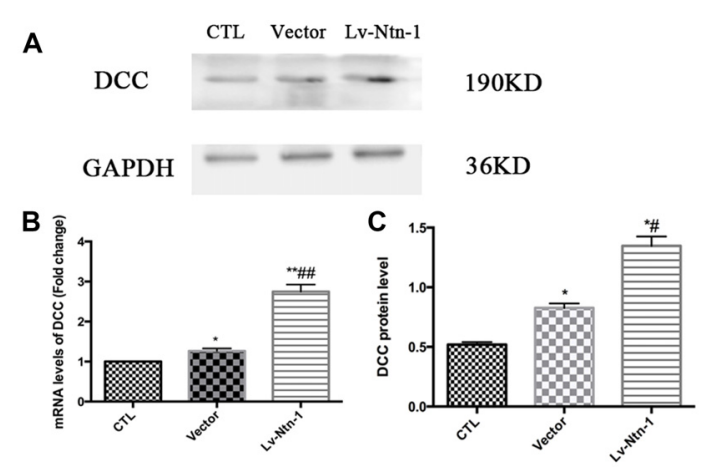

FIGURE 6 | Deleted in colorectal cancer (DCC) was up-regulated by Netrin-1 expression. (A) The image of the detection of DCC by western blot. (B) Analysis of the DCC mRNA levels. Data are presented as mean $\pm \mathrm{SEM}$. ${ }^{*} p<0.05$, as compared with the control group; ${ }^{*} p<0.05$, as compared with the vector group; ${ }^{* *} p<0.01$, as compared with the control group; ${ }^{\# \#} p<0.05$, as compared with the vector group; $n=3$. (C) Analysis of the DCC protein levels. Data are presented as mean \pm SEM. ${ }^{*} p<0.05$, as compared with the control group; ${ }^{\#} p<0.05$, as compared with the vector group; Mann-Whitney U-test; $n=4$.

$6 \mathrm{~h}$ and $12 \mathrm{~h}$ compared with control group (Netrin-1 expression: $1.04 \pm 0.08$ vs. $0.04 \pm 0.01, p<0.05$ and $1.45 \pm 0.03$ vs. $0.04 \pm 0.01, p<0.05$; p-ERK expression: $1.06 \pm 0.11$ vs. $0.43 \pm 0.04, p<0.05$ and $0.83 \pm 0.04$ vs. $0.43 \pm 0.04, p<0.05$ ), but decreased at $24 \mathrm{~h}$ (Figure 1).

\section{Primary Cortical Neurons Were Transfected with Lv-Netrin-1}

Western blot analysis demonstrated that the protein level of Netrin-1 was overexpressed in the Lv-Ntn-1 group when compared with that of the vector group in normal condition and OGD condition (normal condition: $1.66 \pm 0.04$ vs. $0.033 \pm 0.01$, $p<0.05$; OGD condition: $1.76 \pm 0.05$ vs. $0.12 \pm 0.01$, $p<0.05)$. In normal condition, the Netrin-1 expression in the vector group was not significantly increased when compared with the control group $(0.03 \pm 0.003$ vs. $0.03 \pm 0.01$, $p=0.486)$. These results indicate that the transfection of Netrin-1-encoding lentivirus was effective in the primary cortical neurons (Figure 2).

\section{Netrin-1 Protects Primary Cortical Neurons from OGD-Induced Cell Death and Apoptosis}

After the transfection of lentivirus in the cortical neural cells, the effect of Netrin-1 on OGD-induced cell death and apoptosis were further investigated. The death rates of cells after OGD were detected by LDH release, and cell apoptosis was measured by Annexin V-APC/7-AAD. The death rate of primary cortical neurons in the Lv-Nnt-1 group significantly decreased when compared with that of the vector group (5.87 \pm 0.42 vs. $4.30 \pm 0.31, p<0.05)$. The cell apoptosis of the Lv-Nnt1 group also markedly decreased when in comparison with the vector group $(12.73 \pm 0.60 \%$ vs. $27.48 \pm 0.36 \%, p<0.01$; Figure 3).

\section{Netrin-1 Protects Primary Cortical Neurons from OGD Injury via the ERK Pathway}

As reported in existing literature, the activity of ERK pathway was involved in the neuronal apoptosis. Therefore, we speculated whether Netrin-1 affected the activity of ERK. Lentivirus was transfected into different groups. In the Lv-Nnt-1 group, the phosphorylation of ERK was increased in OGD condition but not in normal condition, which indicated that Netrin-1 induced ERK phosphorylation in the primary cortical neurons after OGD $(0.91 \pm 0.032$ vs. $0.57 \pm 0.030, p<0.05)$. U0126, an inhibitor of the Raf/MEK/ERK pathway, was used to mimic the role of ERK pathway in primary cortical neurons after the OGD treatment. After the treatment, the death rate of the Lv-Nnt$1+\mathrm{U} 0126$ group increased significantly when in comparison with that of the Lv-Nnt-1 group ( $4.50 \pm 0.17$ vs. $3.46 \pm 0.25$, $p<0.05$ ) and the apoptosis rate of the Lv-Nnt-1 + U0126 group also elevated when compared with that of the Lv-Nnt-1 group $(28.37 \pm 0.50$ vs. $18.20 \pm 0.72 \%, p<0.001)$. Altogether, these results indicate that Netrin-1 regulates the death rate and apoptosis of primary cortical neurons via the ERK pathway (Figures 4, 5).

\section{Is Up-Regulated by Netrin-1 Expression}

Quantitative real-time PCR was used to measure the mRNA level of DCC. The analysis revealed that the mRNA level of DCC was significantly higher in the Lv-Nnt-1 group when compared with that of the vector group $(2.75 \pm 0.10$ vs. $1.27 \pm 0.04, p<0.001)$. The protein expression of DCC was measured by western blot. As reported, the Lv-Nnt-1 group showed a much higher protein expression than the vector group $(1.35 \pm 0.04$ vs. $0.83 \pm 0.02, p<0.05)$. To confirm the results of western blot, DCC were immunostained in different groups. A larger DCC fluorescence area was evident in the Lv-Nnt-1 group than in the vector and control groups $(3620.96 \pm 149.05$ vs. $2200.65 \pm 97.02$ or $1159.57 \pm 71.69$, $p<0.01)$. As a membrane receptor, the DCC signal was located around the periphery of primary cortical neurons, and DCC signal was weak in control group and vector group (Figures 6, 7).

\section{Anti-DCC Reduces the Phosphorylation of ERK Signaling Pathway}

As DCC and ERK are both involved in the anti-apoptotic effect of Netrin-1, we further investigated whether blocking DCC would affect the ERK signaling. The result of western blot revealed that anti-DCC treatment prevented the activation of ERK signal pathway $(0.50 \pm 0.06$ for the Lv-Nnt-1 group vs. $0.37 \pm 0.02$ for Lv-Nnt-1 + anti-DCC group, $p<0.05)$, suggesting that in the Lv-Nnt-1 group, DCC may play a vital role in decreasing cell death and apoptosis (Figure 8).

\section{Netrin-1 Protects Primary Cortical Neurons by Reducing DNA Damage}

We used comet assay to measure DNA damage. Tail DNA and tail length were detected in different groups. The control group 


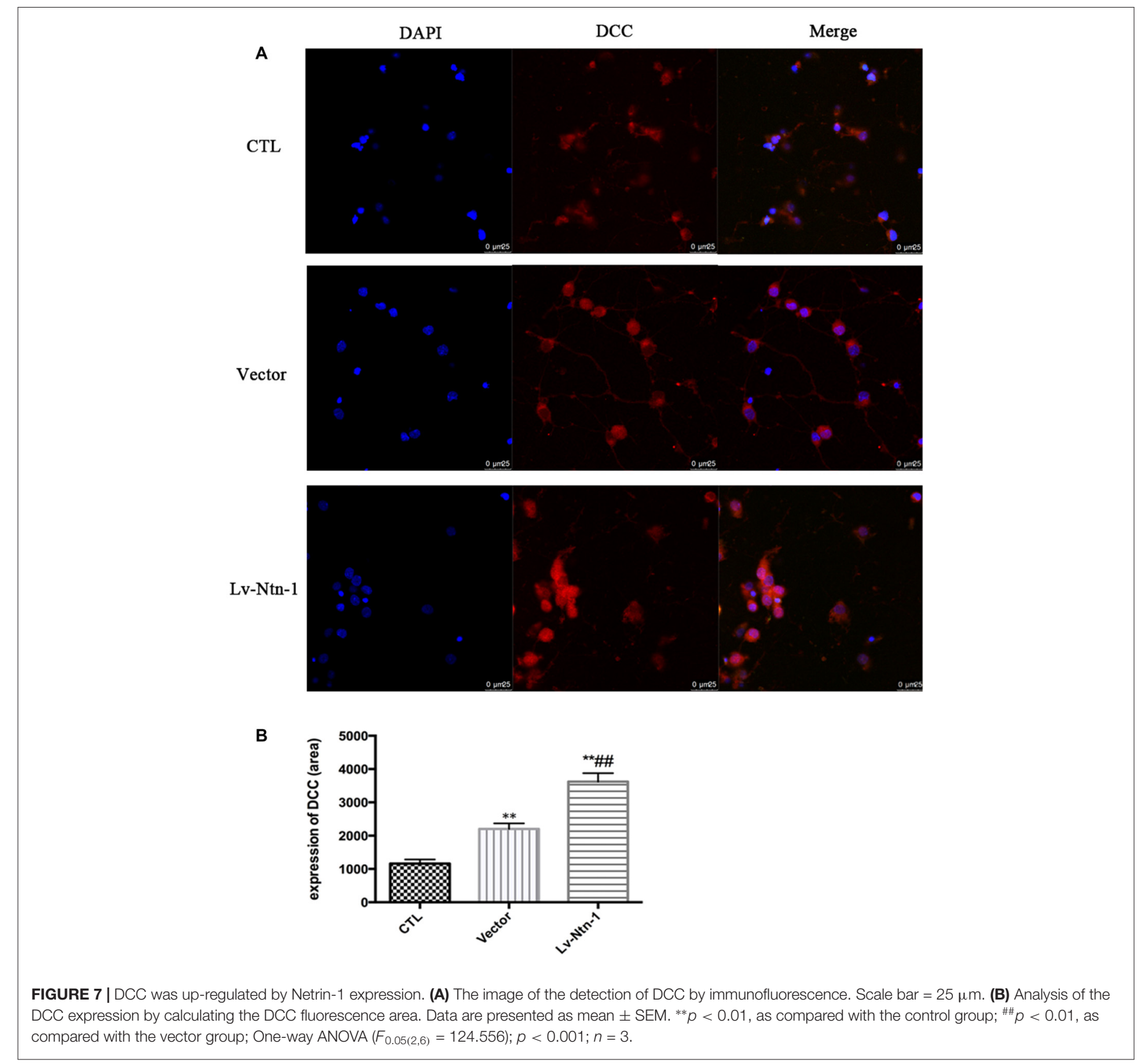

presented a normal nuclear matrix in the nucleus. However, the vector group has a higher level of DNA damage than the Lv-Nnt-1 group (Tail DNA: $13.46 \pm 0.17$ vs. $4.97 \pm 0.18 \%$, $p<0.01$; Tail length: $15.16 \pm 0.37$ vs. $7.90 \pm 0.35, p<0.001$; Figure 9).

\section{DISCUSSION}

In the present study, we speculated whether Netrin-1 could protect neurons from OGD-induced cell death and apoptosis with rat primary cortical neurons. We found that in addition to its role in neuronal migration, Netrin-1 also acted as a survival factor in the cultured primary cortical neurons that were subject to OGD treatment. Moreover, we found that with Netrin-1 overexpression, one of its receptor, DCC, was significantly up-regulated on the protein and mRNA levels, which indicates that Netrin-1 regulates the transcription of DCC. The ERK signaling pathway was involved in the protective effect of Netrin-1 and was activated by Netrin-1 overexpression, which was partly abolished by blocking the receptor DCC. Blocking the ERK signaling with the inhibitor U0126 reversed Netrin-1-induced anti-apoptosis. Netrin-1 protected primary cortical neurons by reducing DNA damage.

Netrin-1 is involved in the survival mechanisms of various human cancer cell lines before it is silenced by siRNA (Delloye- 

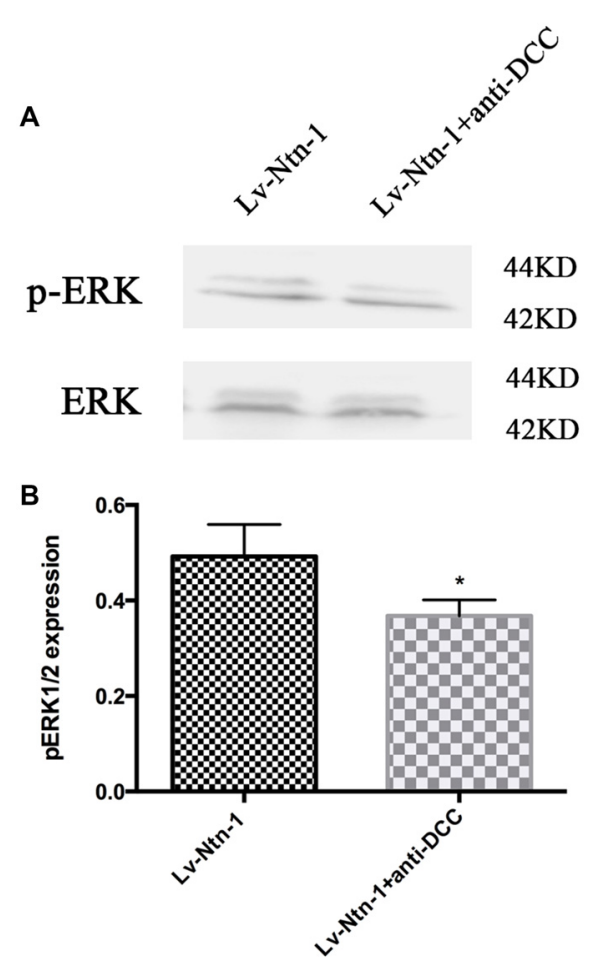

FIGURE 8 | Anti-DCC reduced the phosphorylation of ERK signaling pathway. (A) The image of detection of ERK and phosphorylated ERK. (B) The analysis of $\mathrm{pERK}$ expression by western blot. Data are presented as mean \pm SEM. ${ }^{*} p<0.05$, as compared with the Lv-Ntn-1 group; Mann-Whitney U-test; $n=4$.

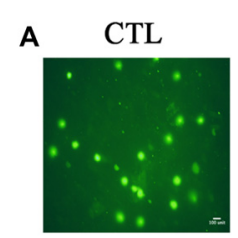

B

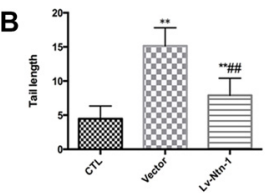

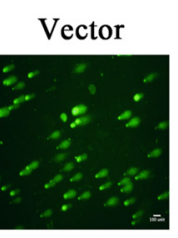

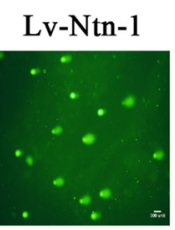

C

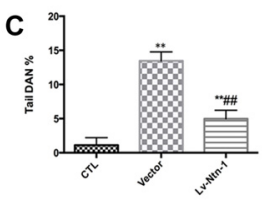

FIGURE 9 | Netrin-1 protected primary cortical neurons by abolishing DNA damage. (A) The image of comet assay. (B) Analysis of the tail length of each group. Data are presented as mean \pm SEM. ${ }^{* *} p<0.01$, as compared with the control group; ${ }^{\#} p<0.01$, as compared with the vector group. (C) Analysis of the tail DNA (\%) of each group. Data are presented as mean \pm SEM. ${ }^{* *} p<0.01$, as compared with the control group; ${ }^{\# \#} p<0.01$, as compared with the vector group; Mann-Whitney $U$-test; $n=3$.

Bourgeois et al., 2009a; Paradisi et al., 2013). Netrin-1 siRNA is associated with a potentiation of cancer cell death in the Doxorubicin treatment, which appears to be p53-dependent (Paradisi et al., 2013). Mazelin et al. (2004) also showed that inhibition of cell death by enforced expression of Netrin-1 in mouse gastrointestinal tract leads to the spontaneous formation of hyperplastic and neoplastic lesions.

In the research of cerebral ischemia, in vivo, Netrin-1 was up-regulated after middle cerebral artery occlusion (Liu et al., 2011). The current study employed an in vitro design and found that Netrin-1 overexpression markedly inhibited neuronal apoptosis when the cultured neurons were subject to OGD condition.

In the model of ischemia/reperfusion-induced myocardial infarction, with Netrin-1 treatment, Netrin-1 receptors were detected, in which the mRNA and protein level of DCC were abundantly elevated (Zhang and Cai, 2010), which is consistent with our research.

In the current study, we found that the ERK signaling pathway was involved in the Netrin-1-induced protection of neurons. The ERK signaling pathway was involved in different pathological conditions (Li et al., 2012; Tan et al., 2013; $\mathrm{Wu}$ et al., 2016). Its activation plays an important role in the proliferation of neural progenitor after cerebral stroke (Kalluri et al., 2007). With the presence of U0126, an inhibitor of $M E K 1 / 2$, the cultured neural progenitors were decreased after OGD compared with the one without U0126. In the research of Chang et al. (2017), the neurite outgrowth was promoted by ARDD-activated ERK phosphorylation, which could be reversed by PD98059, an inhibitor of the ERK pathway. In Sun et al.'s (2017) research, the neuronal apoptosis was significantly affected by NGF/HO-1-activated MEK/ERK pathway after the cultured primary cortical neurons were exposed to OGD.

In our study, Netrin-1 overexpression also activated the ERK signaling pathway, which was partly blocked by blocking the receptor DCC. Blocking the ERK signaling with the inhibitor U0126 reversed the effect of netrin-1-induced anti-apoptosis, which is consistent with the findings reported by the studies mentioned above.

In the research of Zhang and Cai (2010), Netrin-1 protected the heart from myocardial infarction via the activation of the ERK signaling pathway, which was abolished by U0126, indicating that Netrin-1 plays a role in myocardial infarction via the ERK signaling pathway. In his study, the ERK signaling inhibitor reduced DCC expression, indicating a DCC/ERK1/2/eNOSs1177/NO/DCC feed-forward mechanism.

In our study, we found that the activity of ERK signaling pathway was associated with DCC when Netrin-1 was overexpressed. In the analysis, the ERK pathway was inhibited by blocking DCC, but the exact mechanism has not been elucidated yet. In the research of $\mathrm{Ma}$ et al. (2010), there are three conserved sequence motifs, namely, P1, P2 and P3 in cytoplasmic of DCC. The P1 domain of DCC was bound to ERK2 directly, which is in agreement with our result that Netrin-1/DCC complex activates the ERK signaling pathway.

We also found that not only DCC, but the other two receptors of Netrin-1, Unc5B and Unc5C were also increased in the mRNA level after OGD. It remains unclear if Unc5B and Unc5C also participate in the activation of the ERK 
signaling pathway. The ERK signaling pathway was reduced in MiaPaCa II cells with Netrin-1 overexpression (An et al., 2016), which is not consistent with our study. However, in their research, only Unc5B was dramatically increased among the receptors of Netrin-1 and DCC did not increase obviously in $\mathrm{MiaPaCa}$ II cells. We assume that the ERK signaling pathway is activated by DCC instead of Unc5B. There is no evidence that Unc5C is involved in the mediation of the ERK signaling pathway.

In our study, Netrin-1 reduced DNA damage. We speculate that this effect is achieved through the activation of the ERK signaling pathway. As well documented in existing literature, DNA damage can induce cell cycle arrest or apoptosis while the ERK signaling pathway can modulate the cell cycle arrest or apoptosis induced by DNA damage (Lin et al., 2013; Chitikova et al., 2014; Yeh et al., 2014; Liao et al., 2015). A recent study shows that the knockdown of either ERK1 or ERK2 can abolish the Etoposide-induced G2/M arrest (Wei et al., 2010). Another research indicates that the ERK pathway affects the checkpoint activation in DNA damage response, in which the activation of ERK pathway promotes DNA-damage-induced G1/S arrest (Tentner et al., 2012). In addition, the inhibitor of ERK has been reported to reduce the activation of ATM and ATR, two DNA damage response-related kinases (Golding et al., 2007). With the DNA damage, the Double stranded DNA breaks (DSBs) activate DSB repair by homologous recombination (HR). The ERK inhibitor reduces the HR in U87 cells, which demonstrates that the ERK signaling pathway facilitates HR (Wei et al., 2010).

\section{REFERENCES}

An, X. Z., Zhao, Z. G., Luo, Y. X., Zhang, R., Tang, X. Q., Hao, D., et al. (2016). Netrin-1 suppresses the MEK/ERK pathway and ITGB4 in pancreatic cancer. Oncotarget 7, 24719-24733. doi: 10.18632/oncotarget.8348

Chang, S., Ruan, W. C., Xu, Y. Z., Wang, Y. J., Pang, J., Zhang, L. Y., et al. (2017). The natural product 4,10-aromadendranediol induces neuritogenesis in neuronal cells in vitro through activation of the ERK pathway. Acta Pharmacol. Sin. 38, 29-40. doi: 10.1038/aps.2016.115

Chen, C. C., Liu, T. Y., Huang, S. P., Ho, C. T., and Huang, T. C. (2015). Differentiation and apoptosis induction by lovastatin and gamma-tocotrienol in HL-60 cells via Ras/ERK/NF-kappaB and Ras/Akt/NF-kappaB signaling dependent down-regulation of glyoxalase 1 and HMG-CoA reductase. Cell. Signal. 27, 2182-2190. doi: 10.1016/j.cellsig.2015.07.014

Chen, H., Lin, W., Zhang, Y., Lin, L., Chen, J., Zeng, Y., et al. (2016). IL-10 promotes neurite outgrowth and synapse formation in cultured cortical neurons after the oxygen-glucose deprivation via JAK1/STAT3 pathway. Sci. Rep. 6:30459. doi: 10.1038/srep30459

Chitikova, Z. V., Gordeev, S. A., Bykova, T. V., Zubova, S. G., Pospelov, V. A., and Pospelova, T. V. (2014). Sustained activation of DNA damage response in irradiated apoptosis-resistant cells induces reversible senescence associated with mTOR downregulation and expression of stem cell markers. Cell Cycle 13, 1424-1439. doi: 10.4161/cc.28402

Dai, Y., Chen, S., Pei, X. Y., Almenara, J. A., Kramer, L. B., Venditti, C. A., et al. (2008). Interruption of the Ras/MEK/ERK signaling cascade enhances Chk1 inhibitor-induced DNA damage in vitro and in vivo in human multiple myeloma cells. Blood 112, 2439-2449. doi: 10.1182/blood-2008-05159392

Delloye-Bourgeois, C., Brambilla, E., Coissieux, M. M., Guenebeaud, C., Pedeux, R., Firlej, V., et al. (2009a). Interference with netrin-1 and tumor

\section{CONCLUSION}

In cultured primary cortical neurons, Netrin-1 overexpression prevents neurons from apoptosis after OGD via the DCC/ERK pathway, in which Netrin-1 mediates the level and transcription of DCC. In addition, Netrin-1 also reduces the DNA damage of neurons.

\section{AUTHOR CONTRIBUTIONS}

JC drafted the main manuscript and performed the main experiments. HD participated in flow cytometry experiments. NL conceived and designed the experiments. YZ revised the manuscript. PL helped the culture of cortical neurons, western blotting and immunofluorescence staining experiments. JC and $\mathrm{QL}$ took part in $\mathrm{qPCR}$ experiments. RC was responsible for analyzing the data. All authors read and approved the final manuscript.

\section{ACKNOWLEDGMENTS}

This work was supported by the National Natural Science Foundation of China (No. 81472161) and Fujian Province Natural Science Foundation Project (No. 2016B014). The funders had no role in experiment design, data collection and analysis, decision to publish, or preparation of the manuscript. We thank Professor Hongzhi Huang for language revision. We also thank assistant researcher Ding Li from Fujian Medicine University for technical guidance in comet assay.

cell death in non-small cell lung cancer. J. Natl. Cancer Inst. 101, 237-247. doi: 10.1093/jnci/djn491

Delloye-Bourgeois, C., Fitamant, J., Paradisi, A., Cappellen, D., Douc-Rasy, S., Raquin, M. A., et al. (2009b). Netrin-1 acts as a survival factor for aggressive neuroblastoma. J. Exp. Med. 206, 833-847. doi: 10.1084/jem.200 82299

Fan, Y., Shen, F., Chen, Y., Hao, Q., Liu, W., Su, H., et al. (2008). Overexpression of netrin-1 induces neovascularization in the adult mouse brain. J. Cereb. Blood Flow Metab. 28, 1543-1551. doi: 10.1038/jcbfm.2008.39

Forcet, C., Stein, E., Pays, L., Corset, V., Llambi, F., Tessier-Lavigne, M., et al. (2002). Netrin-1-mediated axon outgrowth requires deleted in colorectal cancer-dependent MAPK activation. Nature 417, 443-447. doi: $10.1038 /$ nature748

Furne, C., Rama, N., Corset, V., Chédotal, A., and Mehlen, P. (2008). Netrin-1 is a survival factor during commissural neuron navigation. Proc. Natl. Acad. Sci. U S A 105, 14465-14470. doi: 10.1073/pnas.0803645105

Gao, S., Chen, T., Choi, M. Y., Liang, Y., Xue, J., and Wong, Y. S. (2013). Cyanidin reverses cisplatin-induced apoptosis in HK-2 proximal tubular cells through inhibition of ROS-mediated DNA damage and modulation of the ERK and AKT pathways. Cancer Lett. 333, 36-46. doi: 10.1016/j.canlet.2012. 12.029

Golding, S. E., Rosenberg, E., Neill, S., Dent, P., Povirk, L. F., and Valerie, K. (2007) Extracellular signal-related kinase positively regulates ataxia telangiectasia mutated, homologous recombination repair, and the DNA damage response. Cancer Res. 67, 1046-1053. doi: 10.1158/0008-5472.can-06-2371

Hamasaki, T., Goto, S., Nishikawa, S., and Ushio, Y. (2001). A role of netrin-1 in the formation of the subcortical structure striatum: repulsive action on the migration of late-born striatal neurons. J. Neurosci. 21, 4272-4280.

Harter, P. N., Zinke, J., Scholz, A., Tichy, J., Zachskorn, C., Kvasnicka, H. M., et al. (2014). Netrin-1 expression is an independent prognostic factor for poor 
patient survival in brain metastases. PLoS One 9:e92311. doi: 10.1371/journal. pone.0092311

Kalluri, H. S., Eickstaedt, J., and Dempsey, R. J. (2007). Oxygen glucose deprivation inhibits the growth and ERK phosphorylation of neural progenitor cells in vitro. Neurosci. Lett. 426, 145-148. doi: 10.1016/j.neulet.2007.08.047

Lee, S. J., Lee, S. H., Yoon, M. H., and Park, B. J. (2013). A new p53 target gene, RKIP, is essential for DNA damage-induced cellular senescence and suppression of ERK activation. Neoplasia 15, 727-737. doi: 10.1593/neo. 121862

Lewinska, A., Adamczyk-Grochala, J., Kwasniewicz, E., Deregowska, A., and Wnuk, M. (2017). Diosmin-induced senescence, apoptosis and autophagy in breast cancer cells of different p53 status and ERK activity. Toxicol. Lett. 265, 117-130. doi: 10.1016/j.toxlet.2016.11.018

Li, N., Li, Q., Zhou, X. D., Kolosov, V. P., and Perelman, J. M. (2012). Chronic mechanical stress induces mucin 5AC expression in human bronchial epithelial cells through ERK dependent pathways. Mol. Biol. Rep. 39, 1019-1028. doi: 10.1007/s11033-011-0827-x

Liao, Y., Ling, J., Zhang, G., Liu, F., Tao, S., Han, Z., et al. (2015). Cordycepin induces cell cycle arrest and apoptosis by inducing DNA damage and up-regulation of p53 in Leukemia cells. Cell Cycle 14, 761-771. doi: 10.1080/15384101.2014.1000097

Lin, L., Chen, H., Zhang, Y., Lin, W., Liu, Y., Li, T., et al. (2015). IL10 protects neurites in oxygen-glucose-deprived cortical neurons through the PI3K/Akt pathway. PLoS One 10:e0136959. doi: 10.1371/journal.pone.01 36959

Lin, X., Yan, J., and Tang, D. (2013). ERK kinases modulate the activation of PI3 kinase related kinases (PIKKs) in DNA damage response. Histol. Histopathol. 28, 1547-1554. doi: 10.14670/HH-28.1547

Liu, N., Huang, H., Lin, F., Chen, A., Zhang, Y., Chen, R., et al. (2011). Effects of treadmill exercise on the expression of netrin-1 and its receptors in rat brain after cerebral ischemia. Neuroscience 194, 349-358. doi: 10.1016/j.neuroscience. 2011.07.037

Lu, H., Wang, Y., He, X., Yuan, F., Lin, X., Xie, B., et al. (2012). Netrin1 hyperexpression in mouse brain promotes angiogenesis and long-term neurological recovery after transient focal ischemia. Stroke 43, 838-843. doi: 10.1161/STROKEAHA.111.635235

Ma, W., Shang, Y., Wei, Z., Wen, W., Wang, W., and Zhang, M. (2010). Phosphorylation of DCC by ERK2 is facilitated by direct docking of the receptor P1 domain to the kinase. Structure 18, 1502-1511. doi: 10.1016/j.str. 2010.08.011

Mazelin, L., Bernet, A., Bonod-Bidaud, C., Pays, L., Arnaud, S., Gespach, C., et al. (2004). Netrin-1 controls colorectal tumorigenesis by regulating apoptosis. Nature 431, 80-84. doi: 10.1038/nature02788

Nakka, V. P., Gusain, A., Mehta, S. L., and Raghubir, R. (2008). Molecular mechanisms of apoptosis in cerebral ischemia: multiple neuroprotective opportunities. Mol. Neurobiol. 37, 7-38. doi: 10.1007/s12035-0078013-9

Paradisi, A., Creveaux, M., Gibert, B., Devailly, G., Redoulez, E., Neves, D., et al. (2013). Combining chemotherapeutic agents and netrin-1 interference potentiates cancer cell death. EMBO Mol. Med. 5, 1821-1834. doi: 10.1002/emmm.201302654

Paradisi, A., and Mehlen, P. (2010). Netrin-1, a missing link between chronic inflammation and tumor progression. Cell Cycle 9, 1253-1262. doi: 10.4161/cc. 9.7.11072

Sun, Z., Hu, W., Yin, S., Lu, X., Zuo, W., Ge, S., et al. (2017). NGF protects against oxygen and glucose deprivation-induced oxidative stress and apoptosis by up-regulation of HO-1 through MEK/ERK pathway. Neurosci. Lett. 641, 8-14. doi: 10.1016/j.neulet.2017.01.046

Tan, W. H., Popel, A. S., and Mac Gabhann, F. (2013). Computational model of VEGFR2 pathway to ERK activation and modulation through receptor trafficking. Cell. Signal. 25, 2496-2510. doi: 10.1016/j.cellsig.2013.08.015

Tan, B. B., Zhang, M. M., Li, Y., Zhao, Q., Fan, L. Q., Liu, Y., et al. (2016). Inhibition of Vav3 gene can promote apoptosis of human gastric cancer cell line MGC803 by regulating ERK pathway. Tumour Biol 37, 7823-7833. doi: $10.1007 / \mathrm{s} 13277-015-4505-9$

Tentner, A. R., Lee, M. J., Ostheimer, G. J., Samson, L. D., Lauffenburger, D. A., and Yaffe, M. B. (2012). Combined experimental and computational analysis of DNA damage signaling reveals context-dependent roles for Erk in apoptosis and G1/S arrest after genotoxic stress. Mol. Syst. Biol. 8:568. doi: 10.1038/msb. 2012.1

Thiebault, K., Mazelin, L., Pays, L., Llambi, F., Joly, M. O., Scoazec, J. Y., et al. (2003). The netrin-1 receptors UNC5H are putative tumor suppressors controlling cell death commitment. Proc. Natl. Acad. Sci. US A 100, 4173-4178. doi: 10.1073/pnas.0738063100

Tian, Y., Xie, Q., Tian, Y., Liu, Y., Huang, Z., Fan, C., et al. (2013). Radioactive ${ }^{125} \mathrm{I}$ seed inhibits the cell growth, migration, and invasion of nasopharyngeal carcinoma by triggering DNA damage and inactivating VEGFA/ERK signaling. PLoS One 8:e74038. doi: 10.1371/journal.pone.0074038

Wei, F., Xie, Y., Tao, L., and Tang, D. (2010). Both ERK1 and ERK2 kinases promote G2/M arrest in etoposide-treated MCF7 cells by facilitating ATM activation. Cell. Signal. 22, 1783-1789. doi: 10.1016/j.cellsig.2010.07.007

Weng, L. X., Wang, G. H., Yao, H., Yu, M. F., and Lin, J. (2017). Epigallocatechin gallate inhibits the growth of salivary adenoid cystic carcinoma cells via the EGFR/Erk signal transduction pathway and the mitochondria apoptosis pathway. Neoplasma 64, 563-570. doi: 10.4149/neo_2017_410

Wu, H., Li, G. N., Xie, J., Li, R., Chen, Q. H., Chen, J. Z., et al. (2016). Resveratrol ameliorates myocardial fibrosis by inhibiting ROS/ERK/TGFbeta/periostin pathway in STZ-induced diabetic mice. BMC Cardiovasc. Disord. 16:5. doi: 10.1186/s12872-015-0169-Z

Yeh, Y. T., Yeh, H., Su, S. H., Lin, J. S., Lee, K. J., Shyu, H. W., et al. (2014) Phenethyl isothiocyanate induces DNA damage-associated G2/M arrest and subsequent apoptosis in oral cancer cells with varying p53 mutations. Free Radic. Biol. Med. 74, 1-13. doi: 10.1016/j.freeradbiomed.2014.06.008

Yung, A. R., Nishitani, A. M., and Goodrich, L. V. (2015). Phenotypic analysis of mice completely lacking netrin 1. Development 142, 3686-3691. doi: $10.1242 /$ dev.128942

Zhang, J., and Cai, H. (2010). Netrin-1 prevents ischemia/reperfusion-induced myocardial infarction via a DCC/ERK1/2/eNOS s1177/NO/DCC feed-forward mechanism. J. Mol. Cell. Cardiol. 48, 1060-1070. doi: 10.1016/j.yjmcc.2009. 11.020

Conflict of Interest Statement: The authors declare that the research was conducted in the absence of any commercial or financial relationships that could be construed as a potential conflict of interest.

Copyright () 2017 Chen, Du, Zhang, Chen, Zheng, Lin, Lan, Yuan, Lai, Pan, Chen and Liu. This is an open-access article distributed under the terms of the Creative Commons Attribution License (CC BY). The use, distribution or reproduction in other forums is permitted, provided the original author(s) or licensor are credited and that the original publication in this journal is cited, in accordance with accepted academic practice. No use, distribution or reproduction is permitted which does not comply with these terms. 